\title{
Introduction to insulin pump: part I
}

\section{Commentary}

The incidence of diabetes is reaching alarming numbers all over the world. The patients who require insulin injection for controlling diabetes have two options. Either multiple injections by insulin pen/ syringe or by using the insulin pump. According to comprehensive and well-documented medical researchers, the insulin pump is the best choice to improve blood sugar and protect from developing dangerous complications. Many patients make the transition from multiple injections to the insulin pump. The history of insulin pump goes back in 1963. The first commercial insulin pump was introduced in the USA in 1979. The estimated numbers of home users in the US in 2010 were 500,000 people. The market is more than 4 Billion for the year 2013. The insulin pump is available only by prescription and can be used with Type I, Type II, and gestational diabetes.

The insulin pump is a computerized medical device, which mimics pancreas gland for insulin delivery. It uses only rapid-acting insulin such as Apidra or Novolog, by utilizing basal-bolus insulin delivery. The pump works by delivering background insulin as a basal rate, meal bolus to cover meal's carbohydrates and correction bolus for high blood sugar. Inside of the insulin pump is the motor that delivers insulin. The software is running continuously in the background to check the safety of the pump. The brain of insulin pump holds the proprietary algorithm for calculations, alarms and security checks. Some insulin pumps work wirelessly with blood glucose meter, and the new generation has integrated continuous glucose monitoring system (CGMS). The insulin pumps deliver insulin via infusion set. The infusion set must be replaced every 2-3 days.

Each pump is different by design, pre-loaded proprietary algorithm and, etc. The advance development in medical technology and biomedical industry bring new insulin pumps to the market. Every 2-3 years the insulin pump companies develop upgrade from the previous versions by adding extra features. The new version of insulin pumps with significant changes coming up on the market every 4-6 years.

\section{The major companies on the market for insulin pumps are}

Medtronic is the oldest company in the development of insulin pump and CGMS. The first Insulin pump built by Minimed in 1983. By 2010 total 10 of different versions of insulin pumps were introduced by Minimed The current model is MiniMed 530G with Enlite CGMS, is the 1st insulin pump with the preloaded algorithm to suspend the insulin delivery based on CGMS hypoglycemia alarm. Minimed was the first company that developed CGMS.

Tandem Diabetes Care is the newest insulin pump company which has three models of insulin pumps: $t$ : slim, t: slim+G4 and t: flex. Tandem is the first touchscreen insulin pump available on the market. The technology behind the development of $\mathrm{t}$ : slim insulin pump based on human factor research. Their pump is \#1 for easy and straightforward use.

Animas Company founded in 1996, acquired by Johnson \& Johnson on February 18, 2006. At present time the company has

\author{
Volume 3 Issue 3 - 2016
}

\author{
Eugene Kaganovich \\ Department of Veterans Affairs, VA New York Harbor \\ Healthcare System, USA
}

Correspondence: Eugene Kaganovich, Department of Veterans Affairs, VA New York Harbor Healthcare System, Greater New York City, USA, Email onenotel @gmail.com

Received:September 06, 2016 | Published: September 19, 2016

2 insulin pumps: Animas Ping and new model Animas Vibe with integrated Dexcom G4 platinum sensor. Roche Diagnostic with Accu-Chek Diabetes Care division and their insulin pump Accu-chek combo. The Insulet Corporation founded in 2000. They developed first wireless insulin pump- Omnipod.

\section{The benefits of insulin pump}

a. The essential benefit is to bring A1C below 7\%

b. Sensitive and resistant to insulin

c. Live alone

d. Prevent long-term complication

e. Intensive exercise

f. Insulin stacking

g. Poor glycemic control

h. Frequent or severe hypoglycemia and hypoglycemia unawareness

i. Nighttime hypoglycemia

j. Post meal hyperglycemia

k. Dawn phenomenon

1. Variable work schedule

m. Pregnancy

n. Managing gastroparesis

o. Managing celiac disease by using extended/combo bolus

\section{Drawback}

Every insulin pump is going through FDA approval, which takes a long time. As a result, the pump which FDA approved looks outdated and delayed. The new document from FDA "Infusion Pumps Total Product Life Cycle Guidance for Industry which issued on December 2, 2014, supersedes the "Guidance on the Content of Premarket Notification [510(k)] Submissions for External Infusion Pumps," 
issued March 1993. The FDA regulation and years of waiting for approval push insulin pump technology back. Even after intensive FDA checks on insulin pump recalls still happen.

The most dangerous side effect is diabetic ketoacidosis (DKA) and hypoglycemia. A new era of technological development opens the door to the malicious hacking of wirelessly controlled insulin pumps.

a. Attached to the pump 24/7

b. Weight gain

c. Site Infection

d. Cost

e. Psychological body image barriers

\section{How to choose insulin pump}

a) The first step is to decide if the patient is willing to learn new technology. Agree on checking blood sugar 4-5 times a day, calculate the amount of carbohydrate per meal and closely follow up with the medical team. The insulin pumps are different from each other by the speed of delivery insulin, type of cartridges, infusion sets, and algorithms

b) Speed of bolus delivery

c) The interval of time for basal rate delivery, how many basal rates available to the program

d) Durability, watertight or waterproof

e) User interface (touch screen)

f) How long company is in the business. The insulin pump industry is expanding rapidly. You are as a physician have to choose not only the best pump which will fit the patient demand but also a company with the strong financial background. The companies such as Disetronic (H-Tron, D-Tron insulin pumps), Smith Medical (DeltecCozmo) and recently Asante Snap discontinued production of their pumps g) Available software to upload data from the pump to analyze your settings

h) Do you prefer to have integrated CGMS or not?

i) How much is the cost for upgrade version for the future model

j) Alert, alarms

k) Does insulin pump communicate with blood glucose meter or result must be entered manually?

1) Many patients are going to YouTube video review, where they can see what included with the pump and how to use it. Social media channels are overload with tons of information. How are credible these resources? The wrong information can impact to make proper choices when choosing insulin pump. As a medical professional discuss your concerns, questions with the salesperson who is representing the company.

The insulin pump remains an advance level of diabetes management, and it involves challenges and practical consideration. The medical technology is advancing toward developing the artificial pancreas or close - loop system which requires more complex and significant changes in the insulin pump development and medical management.

\section{Acknowledgments}

To Prof Pablo Caviedes for his constant support and guidance during all my research.

\section{Conflicts of interest}

The author declares there is no conflict of interest. 